\title{
HOMENAJE
}

\section{El aporte científico de Carl Linné a 300 años de su nacimiento}

\author{
The scientific contribution of Carl Linné 300 years after his birth
}

\author{
ARIEL CAMOUSSEIGHT
}

Museo Nacional de Historia Natural, interior Parque Quinta Normal s/n, Casilla 787, Santiago, Chile; e-mail: acamousseight@mnhn.cl

El 23 de mayo se cumplieron 300 años del natalicio de Carl Linné, naturalista sueco, padre de la taxonomía moderna, autor de la nomenclatura binomial para especies. Su aporte a la ciencia fue trascendental y por ello en todo el mundo se está celebrando este aniversario. Revistas como Nature (2007, número 446) han dedicado un número en su honor.

Carl Linné nació en Råshult, en la provincia de Småland en el sudeste de Suecia, el 23 de mayo de 1707. Hijo de padres muy religiosos, Nils Linné, pastor luterano, y Christine Broderson, hija de un pastor. Ello marcó sus creencias. Según sus biógrafos (ChermetteMouratille, 1984), Linné se habría apasionado desde muy pequeño por las plantas. A los siete años ingresó a la escuela de su localidad y a los nueve fue enviado a Växjö, a continuar sus estudios. A esa edad prefería hacer sus propias observaciones de plantas que permanecer en la escuela, por lo que sus camaradas lo apodaban el botánico. A los 15 años conoció a Johan S. Rothman, médico de la región y profesor de la escuela superior, quien lo impulsó a seguir la carrera de medicina en una época en que todos los médicos eran botánicos, pero sus padres lo destinaron a pastor. En 1724, con 17 años, tomó esta última vía, la que pronto abandonó.

En 1727 partió a Lund, donde fue acogido por Kilian Stobaeus (físico). Gracias a su apoyo tuvo acceso a una buena biblioteca que le permitiría instruirse en geología, zoología y, por cierto, botánica. En esta época comenzó sus primeros análisis de clasificación junto con la formación de su herbario. Entre 1728 y 1731 estudió medicina en la Universidad de Uppsala. Allí conoció a Olof Celsius, a quien ayudó a finalizar una obra en dos tomos sobre la flora de la región (Flora upplandica, 1730). Celsius le presentó al profesor Olof Rudbeck, botánico y profesor de medicina, quien apreció sus trabajos, ofreciéndole una plaza de suplente y luego de pasante de sus hijos, acogiéndolo en su casa. Linné le agradeció este apoyo, dedicándole una planta, la Rudbeckia.

Al leer los motivos por los que nombra así la planta, es posible apreciar la fuerza de su creencia religiosa, admiración por la naturaleza y el conocimiento: “... para asegurar la supervivencia de su nombre, hay diversos medios, pero ninguno de ellos permite que esta sea por más de uno o dos siglos... así, queriéndote donar la inmortalidad, sabio Rudbeck, te voy a consagrar una planta y le colocaré tu nombre. Ella bastará para eternizar tu memoria. Tanto tiempo como la tierra exista ... He elegido una planta elevada para recordar tu mérito y los servicios prestados, ... La he querido ramosa y cargada de un gran número de flores y de frutos para mostrar que tú has cultivado a la vez las ciencias y las letras". Linné era creacionista por lo que aceptaba que los animales y las plantas, al haber sido creados por Dios, debían permanecer inmutables en el tiempo.

Durante su primera estadía en Uppsala, a los 23 años de edad, escribió la obra Hortus upplandicus (1730). En ella expuso sus primeras ideas sobre la clasificación de las plantas, siguiendo la distribución y número de sus órganos sexuales reproductivos; para él, los estambres representaban a los hombres y los pistilos a las mujeres, estableciendo de esta forma una analogía con la reproducción humana, cuestión que para los botánicos europeos de la época resultaba chocante, a 
pesar de ello paulatinamente fueron aceptando esta nueva visión de un sistema sexual para clasificar el reino vegetal, reconociendo la utilidad de los órganos considerados en la reproducción pero sin saber de qué manera interactuaban en el proceso, puesto que se desconocía totalmente la fusión de los gametos y el intercambio genético, cuestiones que solo comenzaron a ser visualizadas un siglo después.

Decepcionado por no haber conseguido el puesto dejado por el profesor Rudbeck, abandonó Uppsala y se dedicó a realizar expediciones. Una de las más notables fue a Laponia en 1732, recorriendo en solitario 1.600 $\mathrm{km}$ a pie. De lo observado y recolectado, publicó la obra Florula Laponica (1737), en la que utiliza por primera vez el sistema de clasificación sexual que siete años antes había comenzado a plantear.

En 1735 ingresó a la Universidad de Harderwijk, Holanda, donde desarrolló su trabajo de tesis titulado Praeludia sponsaliorum plantarum (1729) y alcanzó a los 28 años de edad el grado de Doctor en Medicina. Su tesis representa un primer intento en el planteamiento de la taxonomía de las plantas, en la que establece clases en base a los estambres y los órdenes por los pistilos, lo que llevó a determinar, a la luz de los conocimientos actuales, muchas agrupaciones artificiales. Linné vivió y trabajó en Holanda hasta 1738. Visitó Dinamarca, Alemania, Inglaterra y Francia y publicó trabajos, como la primera edición de Systema naturae (1735), Fundamenta botanica (1736), Hortus cliffortianus (1737), Critica botanica (1737), Genera plantarum (1737), Classes plantarum (1738) y la Flora lapponica (1737).

En 1739 regresó a Suecia. Se casó con Sara Lisa Moraea, con quien tuvo seis hijos. Vivió en Estocolmo hasta 1741 trabajando como médico. Regresó a Uppsala después de haber sido nombrado profesor de medicina en esta Universidad, permaneciendo allí por el resto de su vida. Publicó numerosos libros, entre ellos Flora svecica (1745), Fauna svecica (1746) y Philosophia botanica (1751), considerado uno de los más importantes libros en la historia de la sistemática botánica. Dos años más tarde publicó Species plantarum (1753). Esta obra en estricto sentido fue un subproducto de otros estudios, pero es en ella donde comenzó a utilizar formalmente el sistema binomial en la nomenclatura biológica. En la obra, las especies se describen con una frase en latín, limitada a no más de 12 palabras. A estas cortas descripciones o polinomios, Linné las llamó “el nombre propio de cada especie", pero agregó una importante innovación que ya había sido utilizada antes por Caspar Bauhin (1560-1624): la nomenclatura binomial o binomios, como el "nombre corto" de las especies. En los márgenes escribió una sola palabra, la que combinada con la primera palabra del polinomio, formaba un nombre más fácil de recordar y corto para cada especie. Por ejemplo, el pimiento fue nombrado con el polinomio: Schinus folis ferratis impari longiffimo. Al margen aparece la palabra Molle; el nombre corto sería Schinus molle, con el que hasta la fecha se lo conoce.

Para Linné las especies eran las entidades verdaderas que se podían agrupar en categorías más altas llamadas géneros. Esta idea de clasificar en género y especie corresponde a la lógica clásica de Aristóteles, en la que se define por género próximo y diferencia específica. Pero Linné, al instrumentalizar la definición lógica, creó una forma fácil, clara y ordenada para conocer la naturaleza y retener ese conocimiento a través de un nombre. Él afirmaba "nomina si nescis, perit et cognitio rerum", es decir, si ignoras el nombre de las cosas desaparece también lo que sabes de ellas.

En 1758 publicó la décima edición del Sistema naturae, 23 años después de la primera edición, utilizándose por primera vez, de manera consistente, el sistema binomial de los nombres para los animales. Linné postulaba que los estudios de los naturalistas debían intentar revelar el orden divino de la creación, construyendo una clasificación natural que mostrara este orden universal. Por ello, junto con establecer los conceptos de especie y de género, Linné formuló un sistema jerárquico (Sistema naturae), implementado con la creación, además, de órdenes y clases. Cada taxón estaba contenido en el superior, de acuerdo a parecidos anatómicos de estructuras externas (por relaciones fenéticas), proporcionando una imagen estructurada entre las especies. Este conjunto de organismos agrupados según las jerarquías antedichas, constituye el sistema de clasificación de los organismos. 
Con el advenimiento del concepto de evolución (siglo XIX) como explicación de la diversidad biológica y formación de especies (Darwin 1859), aparece la sistemática evolutiva. Posteriormente, con la aparición de la sistemática filogenética (Hennig 1950), se continuó utilizando la clasificación linneana, aunque adaptada para reflejar la filogenia de cada especie, de modo que cada taxón incluya al antecesor y a todos sus descendientes, estructurando lo que actualmente se define como taxón monofilético.

A pesar de los numerosos cambios introducidos al sistema natural (creacionista) de Linné, el actual sistema natural conserva la lógica implementada por este magnífico científico del siglo XVIII, quien desde la observación de la hibridación de las plantas logró vislumbrar la posibilidad de una variación en la inmutabilidad de las especies.

Innumerables son los discípulos y seguidores del sistema de clasificar y nominar a plantas y animales que establece Linné. Entre ellos debemos destacar a Johan Christian Fabricius, naturalista danés (1745-1808), quien en su obra Systema entomologiae (1775) describe las 12 primeras especies de insectos, actualmente aceptadas como originarias del extremo austral de Chile, como por ejemplo Melolontha glacialis Fabricius, Cistela livida Fabricius, Lampyris obscura Fabricius, Aeshna variegata Fabricius, Bombix amphimone Fabricius.
El primer naturalista chileno que emplea para la flora y la fauna de nuestro país el sistema de nomenclatura binaria propuesto por Linné fue Juan Ignacio Molina (1740-1829) (Muñoz 1980). En su obra Saggio sulla storia naturale del Chili (1782) describe, entre otras, cuatro especies de insectos actualmente conocidas como: Epicauta pilme (Molina, 1782), Chylindrophora maulica (Molina), Mathania leucotea (Molina) y Castnia psittacus (Molina).

Carl von Linné, luego de pasar el verano de 1777 en su granja de Hammarby, a 13 kilómetros de Uppsala, falleció el 10 de enero de 1778, a los 71 años. Sus restos fueron enterrados en la catedral de Uppsala. En su honor el rey de Suecia mandó a hacer una medalla con las palabras "priceps botanicorum".

\section{LITERATURA CITADA}

CHERMETTE-MOURATILLE A (1984) Linne, sa vie, son oeuvre. Supplément au Bulletin Mensuel de la Société Linnéenne de Lyon 53: XVII-XXXII.

DARWIN CH (1859) On the origin of species by means of natural selection, or the preservation of favoured races in the struggle for life. John Murray, London, United Kingdom.

HENNIG W (1950) Grundzüge einer Theorie der phylogenetischen Systematik. Berlin, Germany.

MOLINA JI (1782) Saggio sulla storia naturale del Chili. Stamperia di S. Tomase D'Aquino, Bologna, Italia. $367 \mathrm{pp}$.

MUÑOZ PC (1980) El abate Juan Ignacio Molina, botánico. Noticiario Mensual del Museo Nacional de Historia Natural (Chile) 25: 12-16. 


